\title{
Advanced Deep Learning Methods
}

Paul Jäger, Fabian Isensee, Jens Petersen, David Zimmerer, Jakob Wasserthal, Klaus H. Maier-Hein

Division of Medical Image Computing, German Cancer Research Center (DKFZ), Heidelberg, Germany

p.jaeger@dkfz-heidelberg.de

The remarkable rise of deep learning has led to an overwhelming amount of new papers coming up by the week. This tutorial intents to filter out the research most relevant for the medical image computing (MIC) community and present it in a structured and understandable form. It is composed of five parts: Classification, Segmentation, Detection, Generative Models and Semi- Supervised Learning. Each part starts off with a thorough motivation, shows exemplary use cases related to MIC, provides a brief model overview and describes the current state-of-the-art methods in the respective area. Basic knowledge about Neural Networks as covered by the "Deep Learning Fundamentals" tutorial is recommended. 